Abstract CPC-002 Table 1 Qualitative evaluation of pharmacoeconomics study

\begin{tabular}{llllll}
\hline $\begin{array}{l}\text { Economics } \\
\text { Evaluation } \\
\text { T in adjuvant }\end{array}$ & $\begin{array}{l}\text { Study } \\
\text { Design }\end{array}$ & $\begin{array}{l}\text { Data } \\
\text { Collection }\end{array}$ & $\begin{array}{l}\text { Analysis and } \\
\text { Interpretation } \\
\text { of results }\end{array}$ & $\begin{array}{l}\text { Final } \\
\text { Score }\end{array}$ & $\begin{array}{l}\text { Total } \\
\text { relative } \\
\text { score }\end{array}$ \\
\hline Blank & $24 / 26$ & $27 / 45$ & $35 / 48$ & 86 & $72.3 \%$ \\
Chen & $23 / 26$ & $30 / 45$ & $37 / 48$ & 90 & $75.6 \%$ \\
Dedes & $24 / 26$ & $31 / 45$ & $35 / 48$ & 90 & $75.6 \%$ \\
Essers & $24 / 26$ & $30 / 45$ & $35 / 48$ & 89 & $74.8 \%$ \\
Garrison & $24 / 26$ & $31 / 45$ & $36 / 48$ & 91 & $76.5 \%$ \\
Kurian & $25 / 26$ & $31 / 45$ & $37 / 48$ & 93 & $78.1 \%$ \\
Liberato & $26 / 26$ & $33 / 45$ & $39 / 48$ & 98 & $82.3 \%$ \\
Lidgren & $26 / 26$ & $34 / 45$ & $38 / 48$ & 98 & $82.3 \%$ \\
Millar & $25 / 26$ & $32 / 45$ & $35 / 48$ & 92 & $77.3 \%$ \\
Neyt & $25 / 26$ & $31 / 45$ & $34 / 48$ & 90 & $75.6 \%$ \\
NICE & $26 / 26$ & $42 / 45$ & $40 / 48$ & 108 & $90.7 \%$ \\
Norum & $25 / 26$ & $28 / 45$ & $34 / 48$ & 87 & $73.1 \%$ \\
Shiroiwa & $26 / 26$ & $31 / 45$ & $38 / 48$ & 95 & $79.8 \%$ \\
Skedgei & $25 / 26$ & $27 / 45$ & $37 / 48$ & 89 & $74.8 \%$ \\
Van Vlaenderen & $24 / 26$ & $31 / 45$ & $36 / 48$ & 91 & $76.5 \%$ \\
T in MBC & & & & & \\
Poncet & $24 / 26$ & $32 / 45$ & $31 / 48$ & 87 & $73.1 \%$ \\
Norum J & $25 / 26$ & $33 / 45$ & $35 / 48$ & 93 & $78.1 \%$ \\
Elkin & $26 / 26$ & $37 / 45$ & $40 / 48$ & 103 & $86.5 \%$ \\
Lidgren & $26 / 26$ & $36 / 45$ & $38 / 48$ & 100 & $84 \%$ \\
NICE (2002) & $26 / 26$ & $42 / 45$ & $40 / 48$ & 108 & $90.7 \%$ \\
\hline
\end{tabular}

Purpose To evaluate the economic impact of the different prescribing models for trastuzumab on overall costs for breast cancer treatments in the Piemonte Region.

Materials and Methods We systematically reviewed the MEDLINE-indexed, English-language literature to identify published, peer-reviewed economic analyses of trastuzumab in HER2 \pm treatment of breast cancer. We rated study quality as per the Drummond criteria.

Direct medical and unit costs were calculated from the perspective of a Regional health care system. We derived patient data by consulting a Regional administrative database and screening by File F File C and SDO for each patient treated in 2010. To obtain valid data, it is necessary to combine the data from this study into a single model, with an epidemiological measure from the Piemonte Cancer Registry. It is recommended to use an empirical Bayesian analysis to conduct this study because there is no single estimator for the parameters.

Results The search strategy identified 948 articles, of which 340 were citations. From the 608 remaining, 23 articles were considered suitable for full review based on the inclusion criteria. Of these, 15 considered adjuvant trastuzumab treatment only, seven examined metastatic breast cancer treatment and one considered treatment with trastuzumab beyond progression. The analysis of the accuracy of information provided by the information systems showed us that there was only $40 \%$ correspondence with the administrative database within Molinette Hospital.

Conclusions Preliminary results confirm the difficulty of obtaining accurate data from the administrative systems. We hope to obtain precise data on trastuzumab prescribing, and thus offer complementary information to cost-effectiveness analysis before the launching of a generic drug.

No conflict of interest.

\section{CPC-003 A RETROSPECTIVE ANALYSIS OF THE SWITCHES FROM ORIGINATOR AND BIOSIMILAR RECOMBINANT HUMAN ERYTHROPOIETINS IN CHRONIC KIDNEY DISEASE}

doi:10.1136/ejhpharm-2013-000276.460

I Uomo, M Pastorello. Provincial Health Unit Palermo, Department of Pharmacy, Palermo, Italy
Background Erythropoiesis stimulating agents (ESAs) has been shown to be highly effective in anaemia in chronic kidney disease (CKD). Various biological ESAs are available such as epoetin alfa, beta, darbepoetin alfa and C.E.R.A, including three biosimilars, epoetin alfa, zeta and theta. National regulations are trying to promote the prescription of the biosimilars, especially in ESA-naive patients. Switching between products is not recommended and the pharmacist can't replace one epoetin with another. However, changes do occur in clinical practise.

Purpose In the Pharmacy Department of the Palermo Local Health Unit (LHU) we observed that nephrologists frequently switch patients but not in order to reduce costs. Therefore, the aim of this study was to calculate the prevalence and patterns of switching and to evaluate the reasons for them and the results for these changes.

Materials and Methods Distributing all the epoetins, after a discharge or a $\mathrm{DH}$ (docetaxel/trastuzumab) regimen and ensuring appropriate continuity of care, the Department collected and retrospectively analysed an electronic database with all the prescriptions for both non-dialysis-dependent CKD or dialysis patients. Furthermore, haemoglobin levels $(\mathrm{Hb})$ were collected, if available, from the paper prescriptions. The period of observations was January 2011June 2012.

Results 2,711 patients received an epoetin for CKD (from a population of 750,550$) .368$ patients $(13.6 \%)$ had been switched. Of this group, only 194 patients were evaluable (98 female, mean age 73.57+-SD:14.21). The inclusion criteria were: receiving ESAs for at least four months; less than 60 days between two prescriptions. Treatments were less commonly switched from biosimilars than originator formulations. Only in 7 cases did nephrologists cite the lack of efficacy of the ESA previously administered, with demonstrated worsening of the patient's clinical status (Aranesp 4, Mircera 2 and NeoRecormon 1). In 9 cases we assumed lack of efficacy of the first ESA, based on measurement of the haemoglobin $(\mathrm{Hb})$ values. In the following prescription the clinicians switched and reported an $\mathrm{Hb}$ level lower than the first $(\leq 10 \mathrm{~g} / \mathrm{L})$. In 24 cases, the ESAs varied with the prescriber. There was no reason for the switch or it was made for trivial reasons. 5 changes from the biosimilar were the pharmacist's wrong decision, due to not checking the patient's last prescription on the database. 5 changes from Mircera occurred after the announcement of a worldwide shortage. Only in 9 cases had the clinicians decided to shift toward a biosimilar on cost grounds. In the remaining cases, $\mathrm{Hb}$ levels remained stable before and after the switch. We can also state that no spontaneous reports of adverse drug reactions regarding ESAs have been received.

Conclusions Our results demonstrates that all the switches were well tolerated. This may support the use of biosimilars in terms of safety and efficacy and switches towards less expensive epoetins. The decision to start ESA treatment with a biosimilar must be considered, and it will also be possible to change pretreated patients.

No conflict of interest.

\section{CPC-004 A REVIEW OF PHARMACISTSÍ INTERVENTIONS IN A NEUROLOGY DEPARTMENT}

doi:10.1136/ejhpharm-2013-000276.461

PA Jolivot, A Gonthier, E Camps, B Bonan. FOCH hospital, Pharmacy, Suresnes, France

Background The adult Department of Neurology is a 42-bed unit that includes an inpatient neurology ward, an inpatient stroke unit and a 10-bed neurological intensive care unit. The computerised physician order entry system available in our hospital is Omnipro. It enables the pharmacy resident to consult the cause of hospitalisation, nurse care, surveillance of medical parameters and to analyse prescriptions.

Purpose To describe the pharmacists' interventions (PIs). 